\title{
Intraductal temperature-controlled radiofrequency ablation in malignant hilar obstruction: a preliminary study in animals and initial human experience
}

다(1)이우

\author{
Authors

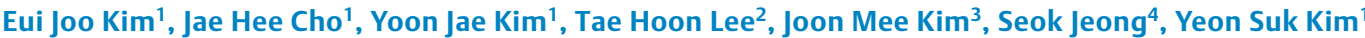

Institutions

1 Department of Internal Medicine, Gachon University College of Medicine, Gil Medical Center, Incheon, Republic of Korea

2 Department of Internal Medicine, Soonchunhyang University College of Medicine, Cheonan Hospital, Cheonan, Republic of Korea

3 Department of Pathology, Inha University School of Medicine, Incheon, Republic of Korea

4 Department of Gastroenterology and Hepatology, and the National Center of Efficacy Evaluation for the Development of Health Products Targeting Digestive Disorders (NCEED), Inha University School of Medicine, Incheon, Republic of Korea

submitted 16.1.2019

accepted after revision 22.5.2019

Bibliography

DOI https://doi.org/10.1055/a-0970-9005 |

Endoscopy International Open 2019; 07: E1293-E1300

(c) Georg Thieme Verlag KG Stuttgart · New York

eISSN 2196-9736

Corresponding author

Jae Hee Cho, MD, PhD, Division of Gastroenterology, Department of Internal Medicine, Gachon University College of Medicine, Gil Medical Center, 21, Namdongdaero 774 beon-gil, Namdong-gu, Incheon, 21565, Republic of Korea

Fax: +82-32-460-3408

jhcho9328@gmail.com

\section{ABSTRACT}

Background and study aims Intraductal radiofrequency ablation (ID-RFA) is a recently developed method widely used for treatment of malignant extrahepatic biliary tract obstructions. However, its safety in hilar application has yet to be clearly demonstrated. The aim of this study was to evaluate the safety of ID-RFA in the treatment of malignant hilar obstruction.

Patients and methods Endoscopic retrograde cholangiography followed by temperature-controlled ID-RFA at the hilar area using different probe lengths $(11,18$, and $22 \mathrm{~mm})$ and settings ( 7 or $10 \mathrm{~W}$ for $60-120 \mathrm{~s}$ ) was performed in six mini-pigs. In addition, patients with malignant hilar obstruction who underwent palliative ID-RFA were retrospectively evaluated.

Results In the animal study using different ID-RFA settings, post-ID-RFA fluoroscopic radiocontrast leakage and microscopic bile duct perforation with hepatic abscess were observed in four of the six mini-pigs. Only two of the them, in which an 11-mm ID-RFA probe at a target temperature of $80^{\circ} \mathrm{C}$, power of $7 \mathrm{~W}$, and duration of $60 \mathrm{~s}$ was used, underwent successful ID-RFA without any immediate adverse events (AEs). Clinically, ID-RFA was performed using the $11-\mathrm{mm}$ probe with the setting of $80^{\circ} \mathrm{C}, 7 \mathrm{~W}$, and $60-120$ s for malignant hilar obstruction, and total of 11 patients underwent successful ID-RFA without AEs.

Conclusions Our study suggests that ID-RFA performed using a short-length probe with settings of $80^{\circ} \mathrm{C}, 7 \mathrm{~W}$ and $60-120 \mathrm{~s}$ is a safe and feasible palliative treatment for malignant hilar obstruction.

\section{Introduction}

Intraductal radiofrequency ablation (ID-RFA) is widely performed for palliation of malignant biliary tract obstruction (MBTO) [1,2], but despite being introduced for clinical use more than 5 years ago, its efficacy is still a matter of debate
[3-7]. Earlier studies included patients with MBTO regardless of the anatomic type, including those with intrahepatic, hilar, and extrahepatic bile duct obstructions $[3,8,9]$. In previous studies that included patients with hilar and/or extrahepatic obstruction, devastating adverse events (AEs) such as perforation, massive hemobilia, and hepatic infarction, especially in patients with Klatskin tumor, were reported [10-13]. 



- Fig. 1 Endobiliary radiofrequency ablation in mini-pig subject. a, b, c, and $\mathbf{d}$ A subject with post-ID-RFA bile duct perforation and contrast leakage. The white arrow indicates the perforation site. $\mathbf{e}, \mathbf{f}, \mathbf{g}$, and $\mathbf{h}$ A subject with successful ID-RFA without any immediate ID-RFA-related adverse event.

In more recent studies, refined inclusion criteria for ID-RFA have taken into account anatomic aspects $[4,8,12,14,15]$. This is of particular relevance given the complicated anatomy of the hilar area, especially the acute angulation along vascular structures. The efficacy and safety of ID-RFA may differ between these patients and those with a distal extrahepatic bile duct; thus, optimal settings of ID-RFA for treatment of hilar malignant obstruction remain to be defined $[16,17]$. A further complication is that temperature-controlled ID-RFA is theoretically different from impedance-controlled ID-RFA, such that specific data are needed to determine optimal settings for each ID-RFA system [18].

In this study, we evaluated safety and optimal settings of temperature-controlled ID-RFA in an in-vivo animal model of hilar malignant obstruction in the presence of a normal bile duct. Clinical feasibility and safety of those settings for palliation in patients with malignant hilar obstruction were also assessed.

\section{Patients and methods}

\section{ID-RFA procedure and instruments}

All ID-RFA procedures were done using an 7Fr (2.31-mm) temperature-controlled ID-RFA catheter (ELRA; STARmed, Goyang, Korea) and RF generator (VIVA Combo; STARmed) under endoscopic retrograde cholangiography (ERC) or percutaneous cholangiography guidance. ID-RFA catheters are manufactured in three forms according to ablation length $(11,18$, or $22 \mathrm{~mm}$ ) and required electrical power is determined by the length of each catheter from 7 to $10 \mathrm{~W}$. The ELRA catheter is based on the temperature-controlled ID-RFA system. If tissue temperature exceeds the preset target temperature, ablation stops, resuming after the tissue temperature drops to below the preset target temperature. Operators can choose catheter length and preset both target temperature and ablation time. After the cholangiogram was acquired, a guidewire was inserted through the hilar area. The ablation catheter was introduced along the guidewire and positioned at the target hilar lesion. After successful ID-RFA, balloon sweeping was done to remove necrotic debris. A post ID-RFA cholangiogram was acquired after the ablation procedure and immediate ID-RFA-related AEs, such as hemobilia and perforation, were evaluated fluoroscopically and endoscopically.

\section{In-vivo animal study}

Six 8-month-old male mini-pigs with normal bile ducts (Cronex Co., Ltd., Hwaseong, Republic of Korea) were used for the invivo animal study. They were kept in pathogen-free animal facilities according to guidelines for use of laboratory animals. ERC was performed using conventional catheters and wires, and was followed by ID-RFA conducted at hilum using different electrical settings, and the three different probes, to determine safe settings for each mini-pig with normal bile ducts ( $>$ Fig. 1; - Table 1). We tested ID-RFA from currently validated ID-RFA settings in extrahepatic bile ducts from maximally invasive settings $\left(22-\mathrm{mm}\right.$ probe, $\left.10 \mathrm{~W}, 80^{\circ} \mathrm{C}, 120 \mathrm{sec}\right)$ to less invasive settings (11-mm probe, $\left.7 \mathrm{~W}, 80^{\circ} \mathrm{C}, 60 \mathrm{sec}\right)[17,19]$. After initial assessment to rule out immediate ID-RFA-related AEs, the procedure was completed without biliary stent insertion. Venous blood samples were taken from the animals before and 24 hours after the procedure. All mini-pigs were euthanized at 24 hours after the procedure, at which time their liver and extrahepatic bile duct were extracted for histological analyses. An experienced clinical pathologist analyzed the extracted specimens and reported their macroscopic and microscopic ID-RFA- 
- Table 1 Summary of in-vivo animal study.

\begin{tabular}{|c|c|c|c|c|c|c|c|c|c|c|}
\hline \multirow[t]{2}{*}{ ID } & \multicolumn{3}{|l|}{ RFA } & \multicolumn{2}{|l|}{ ERC } & \multicolumn{5}{|l|}{ Histology } \\
\hline & $\begin{array}{l}\text { Length } \\
\text { (mm) }\end{array}$ & $\begin{array}{l}\text { Power } \\
\text { (W) }\end{array}$ & $\begin{array}{l}\text { Duration } \\
\text { (sec) }\end{array}$ & $\begin{array}{l}\text { Technical } \\
\text { success }\end{array}$ & $\begin{array}{l}\text { Contrast } \\
\text { leakage }\end{array}$ & $\begin{array}{l}\text { Transmural } \\
\text { necrosis }\end{array}$ & $\begin{array}{l}\text { Microscopic } \\
\text { BD perfora- } \\
\text { tion }\end{array}$ & $\begin{array}{l}\text { Portal } \\
\text { vein } \\
\text { injury }\end{array}$ & $\begin{array}{l}\text { Hepatic } \\
\text { arteryin- } \\
\text { jury }\end{array}$ & $\begin{array}{l}\text { Abscess } \\
\text { formation }\end{array}$ \\
\hline 1 & 22 & $10 \mathrm{~W}$ & 120 & - & + & + & + & + & - & Focal \\
\hline 2 & 18 & $7 W$ & 120 & - & + & + & + & + & - & Large \\
\hline 3 & 18 & $7 W$ & 60 & - & + & + & + & + & + & Large \\
\hline 4 & 11 & $7 W$ & 90 & - & + & + & + & + & + & Large \\
\hline 5 & 11 & $7 \mathrm{~W}$ & 60 & + & - & + & - & - & - & - \\
\hline 6 & 11 & $7 W$ & 60 & + & - & + & - & - & - & - \\
\hline
\end{tabular}

related findings. All procedures were done with the animals under general anesthesia. The Animal Care and Use Committee of our institution reviewed and approved the study (IACUC 17-KE385).

\section{Clinical study}

Based on results obtained from the in-vivo animal study, we retrospectively analyzed the prospectively collected medical records of patients with malignant hilar obstruction requiring palliative endoscopic biliary drainage who underwent ID-RFA at two tertiary medical centers between November 2017 and May 2018. In all patients with newly developed hilar obstruction causing jaundice and/or cholangitis, biliary decompression was accomplished with plastic stent or percutaneous biliary tract drainage.

After confirmation of unresectable malignant hilar obstruction, ID-RFA catheters were selected based on in-vivo data and ID-RFA was performed for 60 to 120 seconds, which was validated in patients with distal MBTO [19]. Inclusion criteria were adult patients with unresectable malignant hilar obstruction with jaundice and/or cholangitis and a requirement for biliary drainage. A self-expanding metal stent (SEMS) or plastic stent was inserted after the ID-RFA procedure, similar to what is done in patients treated with conventional decompression methods at the discretion of expert endoscopists who have more than 10 years of experience in therapeutic ERC. Technical success was defined as successful positioning of ID-RFA probe at the target lesion and ablation using preplanned setting covering the whole length of target malignant stricture. Exclusion criteria were MBTO other than in the hilar area, previous history of ID-RFA and previous SEMS placement for biliary decompression. All patients provided informed consent for ID-RFA. The local hospital ethics committee approved the study protocol (IRB no. GDIRB2018-318).

\section{Statistical analysis}

Statistical analyses were done using SPSS for Windows software (ver. 23.0; IBM Corp., Armonk, New York, United States). Continuous variables are expressed as mean and standard devia- tion, and categorical variables as a number and percentage. Skewed data are reported as the median and range. Student's paired $t$-test was used for analyzing continuous variables with a normal distribution and the Wilcoxon signed rank test was applied for nonparametric paired data. A $P$ value $<0.05$ was considered to indicate statistical significance. An event was defined as re-occlusion of the biliary tract requiring reintervention, or death from any cause. The Kaplan-Meier method was used to estimate event-free survival.

\section{Results}

\section{In-vivo animal study}

Both ERC and ID-RFA were performed successfully in the six treated mini-pigs, and without any difficulty in introducing the catheters and guidewires. The post ID-RFA cholangiogram showed contrast leakage in four of the pigs. In three mini-pigs, a 22-mm or 18-mm ID-RFA probe was used, and in the fourth an 11-mm probe and ablation time of 90 seconds were employed. In two of the technically successful treatments, the procedure was performed using an $11-\mathrm{mm}$ probe and an ablation time of 60 seconds ( $\triangleright$ Table 1 ). Despite the statistically significant increase in white blood cell counts and the blood alanine aminotransferase level, there was no evidence of gastrointestinal bleeding during the 24-hour postoperative period ( $\triangleright$ Table 2 ). All of the animals survived the initial 24 hours postoperatively, at which time they were euthanized for histologic analyses. These revealed transmural necrosis in all six pigs, as well as bile duct perforation with portal vein injury in four of the animals and hepatic artery injury in two. Inflammatory cell infiltration was noted in all of the mini-pigs and gross abscess formation was seen in four ( $>$ Fig. 2 ).

\section{Clinical study}

Between November 2017 and May 2018, 11 patients with malignant hilar obstruction underwent ID-RFA using an 11-mm IDRFA probe, with a target temperature of $80^{\circ} \mathrm{C}$ and an electrical power of $7 \mathrm{~W}$ ( $\triangleright$ Supplementary Table). All patients had hilar obstruction due to an unresectable malignancy, including gall- 
Table 2 Pre- and post procedural lab findings and weight change of mini-pigs.

\begin{tabular}{|c|c|c|c|}
\hline & Pre ID-RFA & 24 hours after ID-RFA & $P$ value \\
\hline \multicolumn{4}{|l|}{ Lab findings, mean (SD) } \\
\hline - WBC, $\times 10^{3}$ cells $/ \mu \mathrm{l}$ & $18.3(2.5)$ & $30.2(6.3)$ & 0.003 \\
\hline - $\mathrm{Hb}, \mathrm{g} / \mathrm{dL}$ & $13.0(0.9)$ & $13.5(1.0)$ & 0.275 \\
\hline - Total bilirubin, g/dL & $0.3(0.0)$ & $0.1(0.0)$ & 0.001 \\
\hline - AST,U/L & $42.3(10.6)$ & $63.7(24.0)$ & 0.096 \\
\hline - $\mathrm{ALT}, \mathrm{U} / \mathrm{L}$ & $46.6(7.1)$ & $50.6(6.9)$ & 0.002 \\
\hline Body weight, kg, mean (SD) & $30.9(0.5)$ & $30.4(0.4)$ & 0.002 \\
\hline
\end{tabular}

ID-RFA, intraductal radiofrequency ablation; WBC, white blood cell; Hb, hemoglobin; AST, aspartate aminotransferase; ALT, alanine aminotransferase


- Fig. 2 Histologic findings of resected specimen from mini-pigs with periductal injury. a RFA applied area reveals severe coagulated necrosis of bile duct without viable structure. b Damaged bile duct shows mucosal coagulative necrosis (black arrow), total lysis of ductal wall (arrow head) and periductal abscess (white arrow). c In some portal veins, neutrophilic phlebitis, endothelial degeneration, and marked intra- and peri-venous hemorrhage are present. $\mathbf{d}$ Adjacent hepatic artery shows prominent neutrophilic infiltration associated with necrosis and hemorrhage of the arterial wall.

bladder cancer, Klatskin tumor, and pancreatic cancer with liver metastasis ( $\vee$ Table 3 ). None of the 11 patients suffered any immediate AEs such as hemobilia or perforation, and the technical success rate was $100 \%(11 / 11)$. Because a relatively short length of ablation was expected using the 11-mm ID-RFA probe, tandem overlapping ID-RFA was attempted in all pa- tients ( $\triangleright$ Fig. 3). Six patients developed post-procedural fever, but all of them recovered fully within 48 hours with conservative management alone. There was no evidence of pneumoperitoneum or hepatic infarction. Median total preoperative serum bilirubin level was $5.8 \mathrm{mg} / \mathrm{dL}$ and it decreased significantly at 24 hours after the procedure $(P=0.008 ; \sim$ Table 4$)$. Median 
- Table 3 Baseline characteristics of included patients.

\begin{tabular}{|l|c|}
\hline Variables & Patients (n=11) \\
\hline Age, mean (SD) & $73.7(7.7)$ \\
\hline Sex, male, $\mathrm{n}(\%)$ & $5(45.5)$ \\
\hline Etiology of hilar obstruction, $\mathrm{n}(\%)$ & \\
\hline - Gallbladder cancer & $2(18.2)$ \\
\hline - Metastatic tumor & $1(9.1)$ \\
\hline - Klatskin tumor & $8(72.7)$ \\
\hline ID-RFA probe length, $\mathrm{n}(\%)$ & \\
\hline - 11 mm & $11(100)$ \\
\hline Ablation power, $\mathrm{n}(\%)$ & $11(100)$ \\
\hline - 7 W & \\
\hline Ablation duration, $\mathrm{n}(\%)$ & $7(63.6)$ \\
\hline - 60 seconds & $4(36.4)$ \\
\hline - 120 seconds & $8(72.7)$ \\
\hline Bilateral stenting, $\mathrm{n}(\%)$ & $4(2-8)$ \\
\hline $\begin{array}{l}\text { Number of overlapping ablation attempts, } \\
\text { median (range) }\end{array}$ & \\
\hline ID-RFA, intraductal radiofrequency ablation & \\
\hline
\end{tabular}

follow-up period was 98 days (range: $30-207$ days) and stent patency was maintained for 30 days in all patients. Three patients required reintervention due to stent occlusion caused by stent ingrowth and/or sludge at 84, 89, and 91 days after the initial procedure, respectively. There were two deaths during the follow-up period due to disease progression; overall survival time of these patients was 151 days and 191 days. Median event-free survival was 91 days (95\% confidence interval: 82 $100)$ according to Kaplan-Meier analysis.

\section{Discussion}

The bile duct is part of the portal triad and enters the liver through invagination of Glisson's capsule at the hilum [20]. The right and left portions of the liver are drained by the right and left intrahepatic ducts, respectively, with acute angulation occurring at their confluence. In the case of hilar malignant obstruction, proximity of the portal triad explains the massive hemobilia or partial hepatic infarction caused by thermal injury to the bile duct, and acute angulation of the confluence accounts for the perforation risk of ID-RFA. Although all of our patients underwent tandem overlapping ablation due to the short length of the ID-RFA catheter, our results support use of a short-length probe as the better option in patients with malignant hilar obstruction. Tumor burden associated with unresectable hilar malignancy may enable a longer ablation time without development of serious AEs. However, considering the anatomic characteristics of the hilar area, a cautious approach is required.
- Table4 Clinical outcome of included patients.

\begin{tabular}{|l|l|}
\hline Variables & Patients (n=11) \\
\hline Technical success, $\mathrm{n}(\%)$ & $11(100)$ \\
\hline Total bilirubin, median (range), $\mathrm{mg} / \mathrm{dL}$ & \\
\hline - At admission & $5.8(2.8-32.1)$ \\
\hline - 1 day after ID-RFA & $2.4(0.6-8.0)$ \\
\hline - 4 weeks after ID-RFA & $1.1(0.5-4.7)$ \\
\hline ID-RFA related early adverse events, $\mathrm{n}(\%)$ & \\
\hline - Perforation & 0 \\
\hline - Hemobilia & 0 \\
\hline - Cholangitis & 0 \\
\hline - Pancreatitis and fever & $1(9.1)$ \\
\hline - Fever only & $5(45.5)$ \\
\hline Total follow-up duration, median (range), $\mathrm{d}$ & $98(30-207)$ \\
\hline - 30-day stent patency, $\mathrm{n}(\%)$ & $11(100)$ \\
\hline - Stent occlusion, $\mathrm{n}(\%)$ & $3(27.3)$ \\
\hline - Mortality during follow-up period, $\mathrm{n}(\%)^{1}$ & $2(18.2)$ \\
\hline ID-RFA, intraductal radiofrequency ablation & \\
\hline Overall survival was 151 days and 191 days for each case. \\
\hline
\end{tabular}

This study demonstrated the safety and feasibility of ID-RFA at the hilar area, based on results obtained in an in-vivo minipig model and a prospectively collected database of ID-RFAtreated patients. The animal study showed that use of a long ID-RFA probe caused bile duct perforation, a finding confirmed by ERC and histologic analyses. As an alternative, use of the short ID-RFA probe for 60 seconds allowed successful hilar ablation in mini-pigs with a normal bile duct. Previous animal studies in which the same temperature-controlled ID-RFA system was used in the distal extrahepatic bile duct reported feasibility of a 33-mm probe at a power of $10 \mathrm{~W}$ and a duration of $120 \mathrm{sec}-$ onds $[17,21]$. However, this setting may not be optimal in humans because the bile duct of mini-pigs differs from that of patients with malignant obstruction. The thickened wall and luminal narrowing characteristic of the malignant obstructive site in humans cannot be reproduced in animal models. However, together with results of previous studies, those of our in-vivo study demonstrate that safety of ID-RFA is influenced by choice of ID-RFA probe and anatomic location of the procedure.

Our study demonstrated the feasibility of ID-RFA performed using an 11-mm RFA probe with settings of $80^{\circ} \mathrm{C}, 7 \mathrm{~W}$ and a duration of 60 to 120 seconds, as in our patients there was no evidence of serious AEs. Although one patient developed mild post-ERCP pancreatitis, it was treated successfully within 48 hours using conservative management only. Incidence of postERCP pancreatitis (9.1\%) was therefore acceptable [22]. Furthermore, based on the ablation site, this AE was probably not ID-RFA-related, but rather ERC-related. 

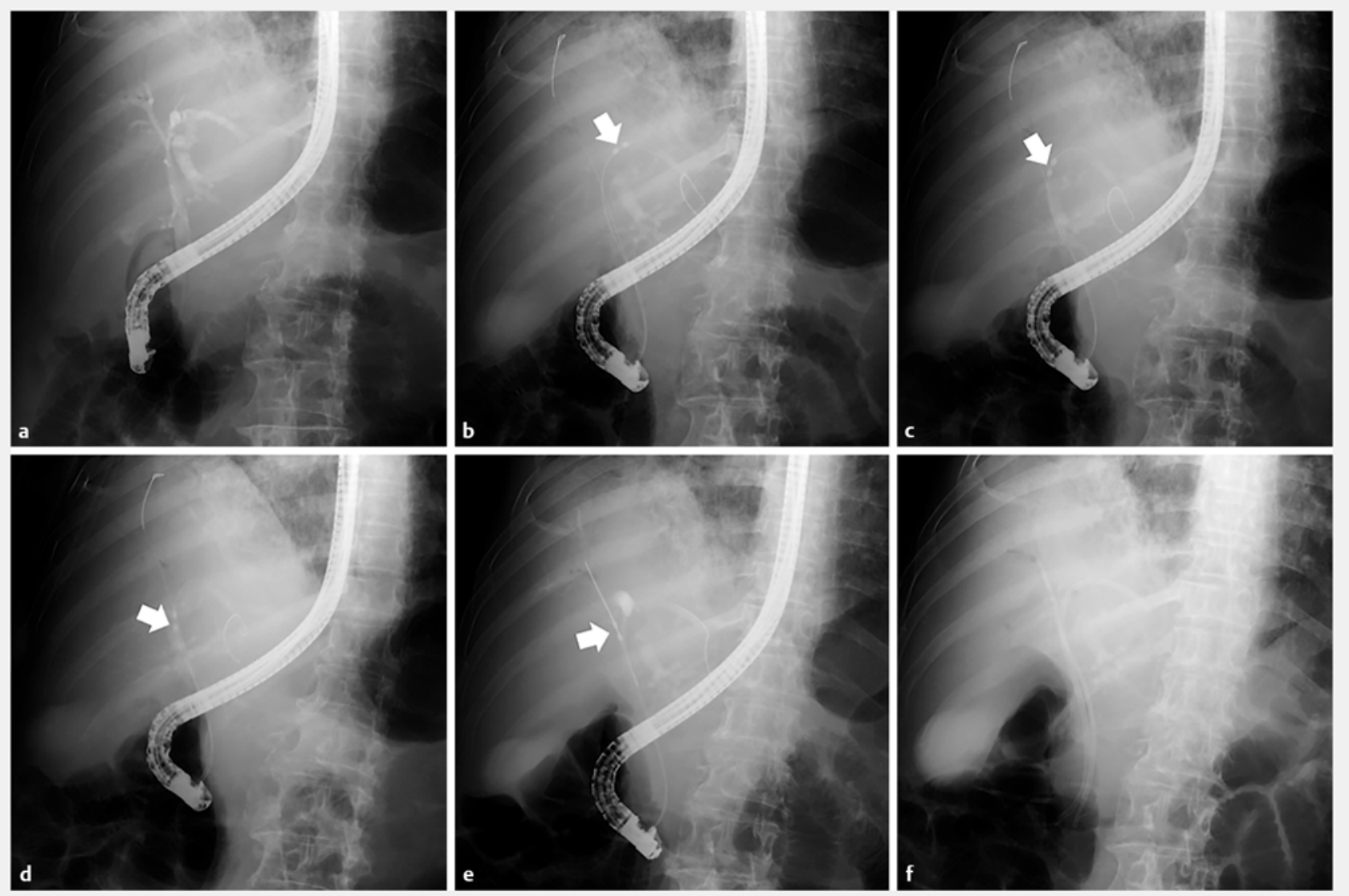

- Fig. 3 Endobiliary radiofrequency ablation in patients with malignant hilar obstruction for palliation. Tandem overlapping ID-RFA using the 11-mm ID-RFA probe was attempted in all patients. The white arrow indicates ID-RFA probe.

The clinical efficacy of ID-RFA with respect to stent patency and overall survival in patients with MBTO has been described in previously published studies [4, 23]. However, most of those studies were limited by a small sample size and retrospective design. Consequently, convincing evidence is lacking for clinical efficacy of ID-RFA for biliary obstruction. A recently published randomized controlled study of distal malignant obstruction suggested use of ID-RFA to improve both stent patency in patients with a plastic stent and overall survival [4]. However, in another study, there was no statistically significant improvement in either stent patency or overall survival in patients who underwent ID-RFA compared to conventional SEMS $[4,5]$. A possible explanation for these discrepant results is heterogeneity of the study population, given both the diverse etiology of MBTO, which includes gallbladder cancer, cholangiocarcinoma, pancreatic cancer and other metastatic malignant diseases, and the small sample sizes [23]. Because the anatomic characteristics of the intrahepatic, hilar, and extrahepatic biliary areas might affect efficacy of ID-RFA, refined strategies and specific clinical settings are likely to be essential for ensuring the success of this procedure. Preprocedural measurement of wall thickness of the target lesion, using intraductal or endoscopic ultrasound, and patient-tailored settings may achieve better clinical outcomes [4]. Further research aimed at determining the optimal patient-tailored settings of ID-RFA is warranted.

Our study of the safety and feasibility of ID-RFA for malignant hilar obstruction had several limitations. It was a singlearm study with a small sample size. Due to the heterogeneous study population, neither overall nor event-free survival could be validated. Thus, settings determined to be optimal in our patients must be evaluated in larger case-control studies, including subgroup analyses, of the clinical efficacy of ID-RFA in terms of stent patency and overall survival.

\section{Conclusion}

ID-RFA using an 11 -mm probe, a target temperature of $80^{\circ} \mathrm{C}$ and a power of $7 \mathrm{~W}$ for a duration 60 seconds was performed safely in the hilar area of mini-pigs with a normal bile duct. In patients with malignant hilar obstruction, a good safety profile of ID-RFA was achieved using a short-length $11 \mathrm{~mm}$ probe with settings of $80^{\circ} \mathrm{C}, 7 \mathrm{~W}$, and 60 to 120 seconds. Further prospective case-control studies are warranted to determine the clinical efficacy of ID-RFA for hilar malignant obstruction based on these safe settings. 


\section{Acknowledgements}

This study was implemented as part of the research project of the Korean Pancreatobiliary Association. This research was supported by Basic Science Research Program through the National Research Foundation of Korea (NRF) funded by the Ministry of Education (grand number: NRF-2018R1D1A3B07041614). Also, this work was supported by the Gachon University Gil Medical Center (grant number: FRD2017-18-02).

\section{Competing interests}

None

\section{References}

[1] Laquiere A, Boustiere C, Leblanc S et al. Safety and feasibility of endoscopic biliary radiofrequency ablation treatment of extrahepatic cholangiocarcinoma. Surg Endosc 2016; 30: 1242 - 1248

[2] Zheng X, Bo ZY, Wan W et al. Endoscopic radiofrequency ablation may be preferable in the management of malignant biliary obstruction: A systematic review and meta-analysis. J Dig Dis 2016; 17 : 716 724

[3] Steel AW, Postgate A], Khorsandi S et al. Endoscopically applied radiofrequency ablation appears to be safe in the treatment of malignant biliary obstruction. Gastrointest Endosc 2011; 73: 149-153

[4] Yang J, Wang J, Zhou $\mathrm{H}$ et al. Efficacy and safety of endoscopic radiofrequency ablation for unresectable extrahepatic cholangiocarcinoma: a randomized trial. Endoscopy 2018; 50: 751 - 760

[5] Teoh AY, Cheung SY, Chong C et al. Endoscopic biliary radiofrequency ablation for malignant distal common bile duct strictures does not improve survival. A randomized controlled trial. Gastrointestinal Endoscopy 2018; 87: AB104-AB105

[6] Wang ], Zhao L, Zhou C et al. Percutaneous intraductal radiofrequency ablation combined with biliary stent placement for nonresectable malignant biliary obstruction improves stent patency but not survival. Medicine (Baltimore) 2016; 95: e3329

[7] Camus M, Napoleon B, Vienne A et al. Efficacy and safety of endobiliary radiofrequency ablation for the eradication of residual neoplasia after endoscopic papillectomy: a multicenter prospective study. Gastrointest Endosc 2018; 88: 511 - 518

[8] Schmidt A, Bloechinger M, Weber A et al. Short-term effects and adverse events of endoscopically applied radiofrequency ablation appear to be comparable with photodynamic therapy in hilar cholangiocarcinoma. United European Gastroenterol J 2016; 4: 570 - 579
[9] Strand DS, Cosgrove ND, Patrie JT et al. ERCP-directed radiofrequency ablation and photodynamic therapy are associated with comparable survival in the treatment of unresectable cholangiocarcinoma. Gastrointest Endosc 2014; 80: $794-804$

[10] Zhou C, Wei B, Gao K et al. Biliary tract perforation following percutaneous endobiliary radiofrequency ablation: A report of two cases. Oncol Lett 2016; 11: 3813-3816

[11] Dolak W, Schreiber F, Schwaighofer H et al. Endoscopic radiofrequency ablation for malignant biliary obstruction: a nationwide retrospective study of 84 consecutive applications. Surg Endosc 2014; 28: $854-860$

[12] Tal AO, Vermehren J, Friedrich-Rust M et al. Intraductal endoscopic radiofrequency ablation for the treatment of hilar non-resectable malignant bile duct obstruction. World J Gastrointest Endosc 2014; 6: $13-19$

[13] Topazian M, Levy M], Patel S et al. Hepatic artery pseudoaneurysm formation following intraductal biliary radiofrequency ablation. Endoscopy 2013; 45: (Suppl. 02): E161 - 162

[14] Wang Y, Cui W, Fan W et al. Percutaneous intraductal radiofrequency ablation in the management of unresectable Bismuth types III and IV hilar cholangiocarcinoma. Oncotarget 2016; 7: $53911-53920$

[15] Wu TT, Li HC, Li WM et al. percutaneous intraluminal radiofrequency ablation for malignant extrahepatic biliary obstruction: a safe and feasible method. Dig Dis Sci 2015; 60: 2158-2163

[16] Wadsworth CA, Westaby D, Khan SA. Endoscopic radiofrequency ablation for cholangiocarcinoma. Curr Opin Gastroenterol 2013; 29: $305-311$

[17] Cho JH, Lee KH, Kim JM et al. Safety and effectiveness of endobiliary radiofrequency ablation according to the different power and target temperature in a swine model. J Gastroenterol Hepatol 2017; 32: $521-526$

[18] Barret M, Leblanc S, Vienne A et al. Optimization of the generator settings for endobiliary radiofrequency ablation. World J Gastrointest Endosc 2015; 7: 1222 - 1229

[19] Kim E], Chung DH, Kim YJ et al. Endobiliary radiofrequency ablation for distal extrahepatic cholangiocarcinoma: A clinicopathological study. PLoS One 2018; 13: e0206694

[20] Castaing D. Surgical anatomy of the biliary tract. HPB (Oxford) 2008; 10: $72-76$

[21] Cho JH, Jeong S, Kim EJ et al. Long-term results of temperature-controlled endobiliary radiofrequency ablation in a normal swine model. Gastrointest Endosc 2018; 87: 1147-1150

[22] Anderson MA, Fisher L. ASGE Standards of Practice Committee. et al. Complications of ERCP. Gastrointest Endosc 2012; 75: 467-473

[23] Sofi AA, Khan MA, Das A et al. Radiofrequency ablation combined with biliary stent placement versus stent placement alone for malignant biliary strictures: a systematic review and meta-analysis. Gastrointest Endosc 2018; 87: 944 - 951 e941 


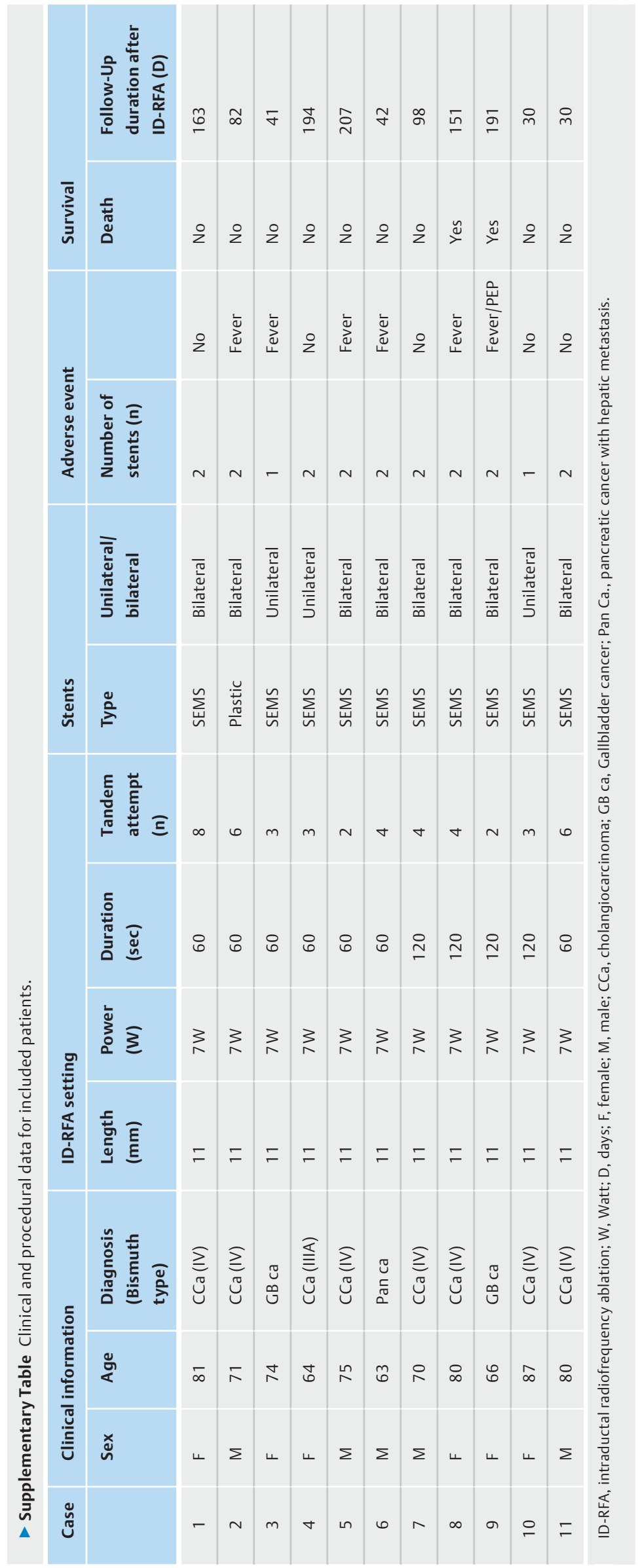

